\title{
Ethnomedicinal Survey of Plants in the Southern District Noakhali, Bangladesh
}

\author{
Rajesh Bhowmik ${ }^{1}$, Monika Rani Saha ${ }^{2}$, Md. Ajijur Rahman ${ }^{2}$ and Md. Anwar Ul Islam² \\ ${ }^{1}$ Department of Pharmacy, Noakhali Science and Technology University, Noakhali, Bangladesh \\ ${ }^{2}$ Department of Pharmacy, University of Rajshahi, Rajshahi-6205, Bangladesh
}

Received: April 06, 2014;

Accepted: June 02, 2014;

Published (Web): July 23, 2014

\begin{abstract}
Many modern medicines used today were discovered by extracting active chemicals from plants used in traditional medicine. To identify and locate any new and potential therapeutic aaplications of commonly used medicinal plants by the folk medicinal practitioners of Noakhali, Bangladesh, we conducted a survery with a semi-structured questionnaire using the guided field-walk method. Detailed information was obtained on 143 plants belonging to 62 families. Leaves were mostly used (52\%) followed by roots (30.55\%) and whole plants (18.74\%) along with fruits, barks, seeds, stems and flowers. Gastrointestinal disorders were the most common diseases treated followed by respiratory tract infections, weakness, fever, diabetes, skin disorders, sexually transmitted diseases, hepatic disorders, pain and rheumatism. Some plants were also used for the treatment of cancer, hypertension, nerve disorders, helmenthiasis, urinary tract infections, tuberculosis, leucorrhea, edema and chicken pox. The findings of this study may help the scientists to get a comprehensive view of the folk medicinal practices in Bangladesh and can use the results to identify medicinal plants of therapeutic interests.
\end{abstract}

Key words: Ethnomedicinal, Traditional medicine, folk medicine, medicinal plants

\section{Introduction}

Medicinal plants play a significant role in the primary healthcare systems for the majority of the rural population (WHO: Traditional medicine; Hamayun et al., 2003). A number of important modern pharmaceuticals have been derived from plants used by indigenous people (Balick and Cox, 1996) including antibiotics, antimalarial drugs, cardiotonics, sympatho and parasympathomimetics etc.The ethomedicinal knowledge about the use of medicinal plants can be a resource for the scientist to identify potential drugs, thus, proper documentation of these knowledges overtime is very essential to protect them from extinction (Cunningham et al., 2002).

In this study, extensive ethnomedicinal surveys have been conducted amongst the folk medicine practitioners with an aim to document ethnomedicinal information of plants used by rural people in various upazila of Noakhali district and to find out any new and unknown therapeutic use of the plants. We have observed considerable variation in the use of medicinal plants by individual Kavirajes (folk medicinal practitioner). There were also many reports of an ethnomedicinal survey conducted amongst folk medicinal practitioners of various district of Bangladesh (Akter et al., 2012; Chowdhury et al., 2011; Das et al., 2012a; Das et al., 2012b; Esha et al., 2012; Hossan et al., 2009a; Hanif et al., 2009b; Mia et al., 2009; Mollik et al., 2009; Mawla et al., 2012; Nawaz et al., 2009a; Nawaz et al., 2009b; Rahmatullah et al., 2009; Rahmatullah et al., 2010a; Rahmatullah et al., 2010b; Rahmatullah et al., 2010c; Rahmatullah et al., 2010d; Rahmatullah et al., 2010f; Rahmatullah et al., 2011; Rahmatullah et al., 2012a; Rahmatullah et al., 2012b; Rana et al., 2010; Shahidullah et al., 2009; Uddin et al., 2004; Uddin et al.,2006). Previously ethnomedicinal surveys were conducted amongst folk medicinal practitioners in some parts of Noakhali and Feni (Rahmatullah, et al., 2011a), here in this survey we have covered all nine upazila of Noakhali district.

\section{Methods}

The survey was conducted in the villages under the different Upazilla (upazila) of Noakhali district namely Begumganj, Maizdi, Senbagh, Chatkhil, Sonaimuri, Kabirhat, Hatiya, Subarnochor and Companigong. A total 
of 20 traditional medical practitioners were interviewed, with their ages ranging between 35 to 70 years and $70 \%$ of them being older than 50 years. Out of the 20 informants interviewed, 18 of them were men and only two were women. The Kavirajes were briefed extensively about the purpose of the visit and told that any information obtained from them may be disseminated in both national and international scientific journals and conferences. Interviews were conducted in the Bangla language (spoken by all Kavirajes) with the help of a semi-structured questionnaire. The basic method followed was that of Martin and Maundu (1995). In this method, the Kavirajes took the interviewers on guided field-walks through areas from where they collected their medicinal plants, pointed out the plants and mentioned their local names, uses and method of use, formulations, diseases for which the formulations were used and dosages. Information was also collected on the season of collection of plants, which plant parts used and whether combination of plants were used to treat any particular disease or if any single plant was used to treat multiple diseases. Data collected from one person were verified with others by asking the same questions.The information reported here is based on the local knowledge.

\section{Results and Discussion}

The study revealed a rich diversity of medicinal plants used to treat various disease conditions of the various upazila in Noakhali district. It was further revealed a rich ethnobotanical knowledge amongst the residents of the various upazila in Noakhali district. The families like Solanaceae, Malvaceae, Rufaceae, Moraceae, Asteraceae, Euphorbiaceae and Cucurbitaceae were the most dominant in this study are consistently recorded in other ethnomedicinal studies.

Table 1. Medicinal plants used for treating human diseases at various upazila in Noakhali.

\begin{tabular}{|c|c|c|c|c|}
\hline Botanical name & Local name & Family & Plant parts used & Uses \\
\hline $\begin{array}{l}\text { Andrographis paniculata } \\
\text { Nees. }\end{array}$ & Kalomegh & Acanthaceae & Leaf & Fever, headache, vertigo. \\
\hline Justicia gendarussa L. & $\begin{array}{l}\text { Bishdorun, Bisa } \\
\text { katail }\end{array}$ & Acanthaceae & Leaf, Root & $\begin{array}{l}\text { Eczema, rheumatism, wounds, } \\
\text { helmenthiases, dysentery, } \\
\text { jaundice. }\end{array}$ \\
\hline Justicia adhatoda $\mathrm{L}$. & Bashok & Acanthaceae & Leaf & $\begin{array}{l}\text { Menstrual problems, asthma, jaundice, } \\
\text { hepatitis. }\end{array}$ \\
\hline Barleria prionitis L. & Jeuti, Jhingti & Acanthaceae & Leaf & Rheumatism, skin disorders, pain. \\
\hline Aptenia cordifolia & Jara lebu & Aizoaceae & $\begin{array}{l}\text { Whole plant, } \\
\text { Fruit }\end{array}$ & $\begin{array}{l}\text { Inflammation, deodorant, headache } \\
\text { poultice. }\end{array}$ \\
\hline $\begin{array}{l}\text { Glinus oppositifolius (L.) } \\
\text { Aug.DC. }\end{array}$ & Tita dima & Aizoaceae & Whole plant & $\begin{array}{l}\text { Skin diseases, earache, indigestion, loss of } \\
\text { appetite. }\end{array}$ \\
\hline Achyranthes aspera $\mathrm{L}$. & Apang & Amaranthaceae & Whole plant & Gonorrhea, leucorrhea. \\
\hline Aerva sanguinolenta $\mathrm{L}$. & Rokto-keshob & Amaranthaceae & Whole plant & Rheumatism, leucorrhea. \\
\hline $\begin{array}{l}\text { Alternanthera sessilis (L.) } \\
\text { DC. }\end{array}$ & Haicha & Amaranthaceae & Leaf & Gonorrhea, leucorrhea. \\
\hline $\begin{array}{l}\text { Lannea grandis (Dennst.) } \\
\text { Engl. }\end{array}$ & Jigar & Anacardiaceae & Meristem, Gum & Venereal diseases. \\
\hline Mangifera indica $\mathrm{L}$. & Aam & Anacardiaceae & Leaf, Seed & Dysentery, helminthiases, liver disorder. \\
\hline Daucus carota L. & Gajor & Apiaceae & Root & Appetite stimulant. \\
\hline Coriandrum sativum. L. & Dhonia & Apiaceae & Seed & Loss of appetite. \\
\hline $\begin{array}{l}\text { Alstonia scholaris (L.) } \\
\text { R.Br. }\end{array}$ & Chatim & Apocynaceae & $\begin{array}{l}\text { Bark, Stem } \\
\text { exudates. }\end{array}$ & Ulcer, diabetes. \\
\hline $\begin{array}{l}\text { Rauwolfia serpentina (L.) } \\
\text { Benth. ex. Kurz. }\end{array}$ & Shorpogonda & Apocynaceae & Whole plant & $\begin{array}{l}\text { Irregular and painful menstration, women } \\
\text { fertility. }\end{array}$ \\
\hline $\begin{array}{l}\text { Alocasia macrorrhizos } \\
\text { (L.) G. Don. }\end{array}$ & $\begin{array}{l}\text { Fenkochu, } \\
\text { Mankochu }\end{array}$ & Araceae & $\begin{array}{l}\text { Root, Stem, } \\
\text { Rhizome }\end{array}$ & Rheumatism, diabetes. \\
\hline $\begin{array}{l}\text { Typhonium giganteum } \\
\text { Engl. }\end{array}$ & Kala kochu & Araceae & $\begin{array}{l}\text { Whole plant, } \\
\text { Stem }\end{array}$ & Stop bleeding, kidney stones. \\
\hline Stendnera virosa Prain. & Bishkochu & Araceae & Root & Rheumatism. \\
\hline
\end{tabular}




\begin{tabular}{|c|c|c|c|c|}
\hline Botanical name & Local name & Family & Plant parts used & Uses \\
\hline $\begin{array}{l}\text { Lasia spinosa }(\mathrm{L} .) \\
\text { Thwaites. }\end{array}$ & Bonadi & Araceae & Leaf, Root & $\begin{array}{l}\text { Joint pain, edema, dysentery, increase } \\
\text { lactation. }\end{array}$ \\
\hline Areca catechu L. & Supari & Arecaceae & Seed & $\begin{array}{l}\text { Carcinogenic, toothache, } \\
\text { addictive. }\end{array}$ \\
\hline Cocos nucifera L. & Narikel & Arecaceae & Leaf, Fruit & Keep head cool, diabetes. \\
\hline $\begin{array}{l}\text { Tylophora indica } \\
\text { (Burm.f.) Merr. }\end{array}$ & Onontomul & Asclepiadaceae & Root & Leucorrhea. \\
\hline $\begin{array}{l}\text { Calotropis gigantean (L.) } \\
\text { R.Br. }\end{array}$ & Apon & Asclepiadaceae & Leaf, Flower & $\begin{array}{l}\text { Fever, rheumatoid arthritis, asthma, } \\
\text { diabetes. }\end{array}$ \\
\hline $\begin{array}{l}\text { Asparagus racemosus } \\
\text { Willd. }\end{array}$ & Shatamuli & Asparagaceae & Bark, Root & $\begin{array}{l}\text { Edema, microbial infections, increases } \\
\text { lactation, weakness. }\end{array}$ \\
\hline Tagetes erecta $\mathrm{L}$. & Gada phool & Asteraceae & Leaf & Stop bleeding and pain. \\
\hline $\begin{array}{l}\text { Mikania cordata (Burm.f.) } \\
\text { B.L. Robinson. }\end{array}$ & Libuji lota & Asteraceae & $\begin{array}{l}\text { Whole plant, } \\
\text { Leaf }\end{array}$ & $\begin{array}{l}\text { Dysentery, diabetes, wounds, gastric } \\
\text { ulcer. }\end{array}$ \\
\hline Blumea lacera DC. & $\begin{array}{l}\text { Hialmutra ,Shiyal- } \\
\text { modi }\end{array}$ & Asteraceae & Leaf,Root & $\begin{array}{l}\text { Weakness, edema, piles, cholera, diuretic, } \\
\text { microbial infections. }\end{array}$ \\
\hline Ageratum conyzoides L. & Ati chada & Asteraceae & Leaf & Diarrhea, boils, skin diseases, joint pain. \\
\hline Basella alba $\mathrm{L}$. & Pui shak & Basellaceae & Leaf, Stem & Anemia, cough. \\
\hline Oroxylum indicum Vent. & Thona & Bignoniaceae & Bark, Seed, Stem & Jaundice, diarrhea, scabies. \\
\hline Bombax ceiba L. & Shimul & Bombacaceae & Root, Fruit & Hypertension. \\
\hline $\begin{array}{l}\text { Ananas comosus (L.) } \\
\text { Merr. }\end{array}$ & Anarosh & Bromeliaceae & Leaf & Jaundice, helminthiasis. \\
\hline Carica papaya $\mathrm{L}$. & Papae & Caricaceae & Fruit & $\begin{array}{l}\text { Indigestion, jaundice, toothache, kidney } \\
\text { stones. }\end{array}$ \\
\hline Terminalia chebula Retz. & Haritaki & Combretaceae & Bark & Stomachic, acidity. \\
\hline $\begin{array}{l}\text { Terminalia arjuna (Roxb. } \\
\text { ex DC.) Wight \& Arn. }\end{array}$ & Arjun & Combretaceae & Bark & $\begin{array}{l}\text { Asthma, dysentery, heart disorder, sexual } \\
\text { diseases. }\end{array}$ \\
\hline $\begin{array}{l}\text { Terminalia belerica } \\
\text { (Gaertn.) Roxb. }\end{array}$ & Bohera & Combretaceae & Fruit & Asthma, allergy. \\
\hline $\begin{array}{l}\text { Commelina benghalensis } \\
\text { L. }\end{array}$ & Kola duma & Commelinaceae & Leaf, Root & External poisoning, burns. \\
\hline Cuscuta reflexa Roxb. & Swarnalata & Convolvulaceae & Leaf, Stem & Hypertension, skin disease, liver cirrhosis \\
\hline Ipomoea aquatica Forssk. & Kalmi lota & Convolvulaceae & Leaf & Gastric and intestinal disorders, diabetes. \\
\hline $\begin{array}{l}\text { Kalanchoe pinnata (Lam.) } \\
\text { Pers. }\end{array}$ & Pathorkuchi & Crassulaceae & Leaf & $\begin{array}{l}\text { Urinary problems, kidney or gall bladder } \\
\text { stones. }\end{array}$ \\
\hline Raphanus sativus L. & Mula & Cruciferae & Root & Increase digestion, decreases acidity. \\
\hline Momordica charantia $\mathrm{L}$. & Korolla & Cucurbitacea & Leaf & $\begin{array}{l}\text { Diabetis, helminthiasis, jaundice, ulcer, } \\
\text { constipation. }\end{array}$ \\
\hline Citrullus lanatu (Thunb.) & Tormuj & Cucurbitaceae & Fruit & Heart disorders, typhoid fever. \\
\hline $\begin{array}{l}\text { Cucurbita maxima } \\
\text { Duchesne. }\end{array}$ & Mishti kumra & Cucurbitaceae & Fruit & $\begin{array}{l}\text { Gastrointestinal problems, joint pain, } \\
\text { piles. }\end{array}$ \\
\hline $\begin{array}{l}\text { Lagenaria siceraria } \\
\text { (Molina) Standl. }\end{array}$ & Lau & Cucurbitaceae & Fruit & Keep head cool, cholera. \\
\hline $\begin{array}{l}\text { Momordica } \\
\text { cochinchinensis (Lour.) } \\
\text { Spreng. }\end{array}$ & Kakroal & Cucurbitaceae & Fruit & Diabetes. \\
\hline Cyperus rotundus $\mathrm{L}$. & Mutha & Cyperaceae & Root & $\begin{array}{l}\text { Weakness, fever, diarrhea, wound, } \\
\text { cholera, vomiting. }\end{array}$ \\
\hline Ricinus communis $\mathrm{L}$. & Arondo mul & Euphorbiaceae & Leaf, Seed & $\begin{array}{l}\text { Gastric distress, liver or gall bladder } \\
\text { disorder, constipation, headache, joint } \\
\text { pain. }\end{array}$ \\
\hline $\begin{array}{l}\text { Phyllanthus fraternus } \\
\text { G.L.Webster. }\end{array}$ & Vui amloki & Euphorbiaceae & $\begin{array}{l}\text { Whole plant, } \\
\text { Root, Leaf }\end{array}$ & $\begin{array}{l}\text { Fever, joint pain, gonorrhea, diarrhea, } \\
\text { liver or gall bladder disorder. }\end{array}$ \\
\hline Phyllanthus emblica & Amloki, Amla & Euphorbiaceae. & Bark, Fruit & Diabetes, dysentery, venereal diseases. \\
\hline
\end{tabular}




\begin{tabular}{|c|c|c|c|c|}
\hline Botanical name & Local name & Family & Plant parts used & Uses \\
\hline $\begin{array}{l}\text { Euphorbia prostrata } \\
\text { Aiton. }\end{array}$ & Khir-kakoli & Euphorbioaceae & Whole plant & Piles, hemorrhoids. \\
\hline Mimosa pudica $\mathrm{L}$. & Lojjaboti & Fabaceae & $\begin{array}{l}\text { Whole plant, } \\
\text { Root }\end{array}$ & $\begin{array}{l}\text { Piles, wound, dysentery, prevent decaying } \\
\text { of gums, urine tract stone, skin diseases. }\end{array}$ \\
\hline Tamarindus indica $\mathrm{L}$. & Tetul & Fabaceae & Leaf, Fruit & $\begin{array}{l}\text { Cold, dysentery, rheumatic pain, burning } \\
\text { sensations in hands or feet, oral lesions. }\end{array}$ \\
\hline Convolvulus & Shonkapuspo & Gentianaceae & Leaf, Flower & Tumor, anxiety, neurosis, epilepsy. \\
\hline \multicolumn{5}{|l|}{ Pluricaulis } \\
\hline Leonurus sibiricus L. & Raktodrone & Labiatae & Leaf, Root & Cancer, helminthiasis. \\
\hline Ocimum sanctum. L. & Tulsi & Lamiaceae & Leaf & Fever, cough, indigestion. \\
\hline Hyptis suaveolens Poit. & Tokma & Lamiaceae & Seed, Leaf & Gonorrhea, microbial infections. \\
\hline Leucas aspera Willd. & Dron pushpo & Lamiaceae & $\begin{array}{l}\text { Leaf, Stem, } \\
\text { Flower }\end{array}$ & $\begin{array}{l}\text { Eye inflammation, external bleeding and } \\
\text { dysentery. }\end{array}$ \\
\hline $\begin{array}{l}\text { Barringtonia acutangula } \\
\text { (L.) Gaertn. }\end{array}$ & Hijal & Lecythidaceae & Leaf, Seed, Root & Diarrhea, headache, chest pain. \\
\hline $\begin{array}{l}\text { Clitorea tarretea }(\mathrm{L} .) \\
\text { Gaertn. }\end{array}$ & Aparajita Nila & Leguminosae & Leaf & Eye disease, cataract. \\
\hline Cassia alata (L.) Gaertn. & Daud pata & Leguminosae & Leaf & Skin diseases, microbial infections. \\
\hline Cajanus cajan (L.) Millsp. & Aral pata & Leguminosae & Leaf, Seed & $\begin{array}{l}\text { Jaundice, helminthiasis, lower cholesterol } \\
\text { level, diabetes. }\end{array}$ \\
\hline Cassia fistula $\mathrm{L}$. & Sonalu & Leguminosae & $\begin{array}{l}\text { Leaf, Bark, Root, } \\
\text { Fruit }\end{array}$ & Helminthiasis, constipation. \\
\hline $\begin{array}{l}\text { Pterocarpus santalinus } \\
\text { L.f. }\end{array}$ & Roktochondon & Leguminosae & Bark & $\begin{array}{l}\text { Tuberculosis, debility, burning sensations } \\
\text { during urination. }\end{array}$ \\
\hline Dalbergia sisso Roxb. & Shishu & Leguminosae & Leaf, Root & Inflammation, astringent. \\
\hline $\begin{array}{l}\text { Saraca asoca (Roxb.) de } \\
\text { Wilde. }\end{array}$ & Ashok & Leguminosae & Leaf, Bark & Dysentery, irregular menstration, piles. \\
\hline Abrus precatorius $\mathrm{L}$. & Jastimadhu & Leguminosae & Root & Debility, fever, cough. \\
\hline Erythrina variegate L. & Mandar & Leguminosae & Leaf, Seed, & Piles, asthma, epilepsy, astringent. \\
\hline Allium cepa $\mathrm{L}$. & Piyaaz & Liliaceae & Seed & $\begin{array}{l}\text { Dandruff, hair loss, insect bite, induces } \\
\text { sleep. }\end{array}$ \\
\hline Allium sativum. L. & Roshun & Liliaceae & Bulb & Cold, hair loss, diabetes, helminthiasis. \\
\hline Aloe barbadensis Mill. & Ghritokumari & Liliaceae & Leaf & $\begin{array}{l}\text { Diabetes, constipation, asthma, flatulance, } \\
\text { hair tonic, burning sensation during sexual } \\
\text { ejaculation. }\end{array}$ \\
\hline $\begin{array}{l}\text { Lygodium } \\
\text { Flexuosum (L.) Sw. }\end{array}$ & Dheki shak & Lygodiaceae & $\begin{array}{l}\text { Whole plant, } \\
\text { Root, Leaf }\end{array}$ & Rheumatism, eczema, cough, wounds. \\
\hline Punica granatum. L. & Dalim & Lythraceae & Leaf, bark & $\begin{array}{l}\text { Inflammation, heart disorder, loss of } \\
\text { appetite, blood dysentery. }\end{array}$ \\
\hline Lawsonia inermis $\mathrm{L}$. & Mehandi & Lythraceae & Leaf & Keep head cool, dandruff. \\
\hline Hibiscus mutabilis L. & Sthol-padma & Malvaceae & Leaf & Reduce burning sensations in the body. \\
\hline Abroma augusta & Ulot kombol & Malvaceae & Bark, Root & Dysmenorrhea, diabetes. \\
\hline Hibiscus rosa sinensis L. & Roktojoba & Malvaceae & Leaf, Flower & Dysentery, leucorrhea. \\
\hline $\begin{array}{l}\text { Malvaviscus arboreus } \\
\text { Dill. ex Cav. }\end{array}$ & Morich-phool & Malvaceae & Leaf, Flower & Skin diseases. \\
\hline Sida rhombifolia L. & Bailodi & Malvaceae & Leaf, Stem Root & Rheumatic pain. \\
\hline Urena lobata $\mathrm{L}$. & Bilai-kan & Malvaceae & $\begin{array}{l}\text { Leaf, Root, } \\
\text { Flower }\end{array}$ & $\begin{array}{l}\text { Malaria, gonorrhea, wound, toothache, ear } \\
\text { infections. }\end{array}$ \\
\hline $\begin{array}{l}\text { Aphanamixis polystachya } \\
\text { (Wall.) R. Parker. }\end{array}$ & Pitraj & Meliaceae & $\begin{array}{l}\text { Stem, Leaf, } \\
\text { Bark }\end{array}$ & Cancer, insecticide. \\
\hline $\begin{array}{l}\text { Azadirachta indica A. } \\
\text { Juss. }\end{array}$ & Neem & Meliaceae & $\begin{array}{l}\text { Leaf, Bark, } \\
\text { Seed }\end{array}$ & $\begin{array}{l}\text { Diabetes, measles, pox, scabies, } \\
\text { indigestion, cataract, abscess. }\end{array}$ \\
\hline $\begin{array}{l}\text { Tinospora sinensis (Lour.) } \\
\text { Merrill. }\end{array}$ & Guloncho & Menispermaceae & Stem & $\begin{array}{l}\text { Tuberculosis, debility, burning sensations } \\
\text { during urination. }\end{array}$ \\
\hline
\end{tabular}




\begin{tabular}{|c|c|c|c|c|}
\hline Botanical name & Local name & Family & Plant parts used & Uses \\
\hline $\begin{array}{l}\text { Stephania japonica } \\
\text { (Thunb. ex Murray) } \\
\text { Miers. }\end{array}$ & Modiani, mucchani & Menispermaceae & $\begin{array}{l}\text { Whole plant, } \\
\text { Leaf, Root }\end{array}$ & Fever, diarrhea, urinary diseases. \\
\hline $\begin{array}{l}\text { Artocarpus heterophyllus } \\
\text { Lam. }\end{array}$ & Kathal & Moraceae & $\begin{array}{l}\text { Leaf, Fruit, } \\
\text { Seed }\end{array}$ & $\begin{array}{l}\text { Ulcer, constipation, diuretic, carminative, } \\
\text { diarrhea. }\end{array}$ \\
\hline Ficus benghalensis L. & Bot, Bar & Moraceae & $\begin{array}{l}\text { Bark, Root, } \\
\text { Leaf, Seed }\end{array}$ & Piles, astringent, aphrodisiac. \\
\hline Ficus racemosa $\mathrm{L}$. & Jogh-dumur & Moraceae & $\begin{array}{l}\text { Fruit, Bark, } \\
\text { Root, Fruit }\end{array}$ & Leucorrhea, diabetes, piles, astringent. \\
\hline Ficus religiosa L. & Ashoth & Moraceae & Bark, Leaf, Fruit & Gonorrhea, constipation, astringent. \\
\hline Streblus asper Lour. & Hapra & Moraceae & Whole plant & $\begin{array}{l}\text { Fever, chicken pox, diarrhea, rheumatic } \\
\text { fever. }\end{array}$ \\
\hline Moringa oleifera & Sajna & Moringaceae & Bark, Fruit & Hypertension, indigestion. \\
\hline Psidium guajava $\mathrm{L}$. & Piyara & Myrtaceae & Leaf & $\begin{array}{l}\text { Diarrhea, menstrual problems, tooth } \\
\text { infections. }\end{array}$ \\
\hline Boerhaavia diffusa $\mathrm{L}$. & Punarnava & Nyctaginaceae & $\begin{array}{l}\text { Leaf, Root, } \\
\text { Stem }\end{array}$ & $\begin{array}{l}\text { Jaundice, anemia, gonorrhea, abdominal } \\
\text { pain, dysentery, elephantiasis. }\end{array}$ \\
\hline Nyctanthes arbor tristis L. & Shefali, Sheuli & Oleaceae & Leaf, Bark & Fever, rheumatism, cough. \\
\hline $\begin{array}{l}\text { Ludwigia hyssopifolia }(\mathrm{G} \\
\text { Don) Exell. }\end{array}$ & At-tika & Onagraceae & Stem & Liver or gall bladder disorders. \\
\hline Ludwigia prostrata Roxb. & $\begin{array}{l}\text { Kalo-keshari, agni- } \\
\text { dolon }\end{array}$ & Onagraceae & Whole plant & Abscess, leucorrhea. \\
\hline $\begin{array}{l}\text { Rhynchostylis retusa } \\
\text { Blume. }\end{array}$ & Rashna & Orchidaceae & Leaf, Root & $\begin{array}{l}\text { Rheumatic pain, wound, fungus } \\
\text { infections. }\end{array}$ \\
\hline Pandanus foetidus Roxb. & Keya kanta & Pandanaceae & Root & $\begin{array}{l}\text { Diarrhea, central nervous system } \\
\text { stimulant. }\end{array}$ \\
\hline Piper nigrum L. & Gol morich & Piperaceae & Fruit, Seed & $\begin{array}{l}\text { Chest and joint pain, dysentery, hair loss, } \\
\text { frequent urination. }\end{array}$ \\
\hline Piper longum L. & Pipul & Piperaceae & $\begin{array}{l}\text { Whole } \\
\text { plant,Fruit }\end{array}$ & $\begin{array}{l}\text { Tuberculosis, asthma, bronchitis, } \\
\text { indigestion. }\end{array}$ \\
\hline Piper betle L. & Paan pata & Piperaceae & Leaf & $\begin{array}{l}\text { Stomach disorders, breast and prostate } \\
\text { cancer. }\end{array}$ \\
\hline $\begin{array}{l}\text { Piper chaba } \\
\text { W. Hunter. }\end{array}$ & Bon pipul & Piperaceae & $\begin{array}{l}\text { Whole plant, } \\
\text { Fruit, Root }\end{array}$ & Weakness, asthma, rheumatic pain. \\
\hline Piper cubeba L.f. & Kabab-chini & Piperaceae & Whole plant & Irregular and painful menstruation. \\
\hline Plumbago zeylanica $\mathrm{L}$. & Chitamool & Plumbaginaceae & Root & Ulcer, gastrointestinal disorder, antiseptic. \\
\hline Oryza sativa $\mathrm{L}$. & Dhan pata & Poaceae & Leaf & Piles, loss of appetite, scurvey, flatulence. \\
\hline $\begin{array}{l}\text { Bambusa arundinacea } \\
\text { (Retz.) Willd. }\end{array}$ & Bash & Poaceae & $\begin{array}{l}\text { Leaf,Stem, } \\
\text { Root }\end{array}$ & Cough, asthma, joint pain, stops bleeding. \\
\hline $\begin{array}{l}\text { Cynodon dactylon (L.) } \\
\text { Pers. }\end{array}$ & Durba & Poaceae & Whole plant & $\begin{array}{l}\text { Piles, bronchitis, flatulence, loss of } \\
\text { appetite. }\end{array}$ \\
\hline Saccharum spontaneum L. & Moyda & Poaceae & Whole plant & Vomiting. \\
\hline Rosa damascene Mill. & Golap phool & Rosaceae & Leaf, Flower & Menstrual problems, skin spots. \\
\hline Paederia foetida $\mathrm{L}$. & Gondho badali & Rubiaceae & Leaf,Root, Fruit & $\begin{array}{l}\text { Indigestion, weakness, cancer, toothache, } \\
\text { dysentery. }\end{array}$ \\
\hline $\begin{array}{l}\text { Hedyotis corymbosa }(\mathrm{L} .) \\
\text { Lam. }\end{array}$ & Khetroa & Rubiaceae & Whole plant & $\begin{array}{l}\text { Jaundice, liver disorders, fever, heat } \\
\text { eruptions. }\end{array}$ \\
\hline $\begin{array}{l}\text { Citrus aurantiifolia } \\
\text { (Christm.) Swingle. }\end{array}$ & Batabi lebu & Rutaceae & Fruit & $\begin{array}{l}\text { Diabetes, indigestion, insect bite, oral } \\
\text { lesions, increases eye sight. }\end{array}$ \\
\hline Citrus aurantium $\mathrm{L}$. & Komla & Rutaceae & Skin of fruit & Diarrhea. \\
\hline Citrus acida Roxb. & Lebu & Rutaceae & $\begin{array}{l}\text { Fruit, Skin of } \\
\text { Fruit }\end{array}$ & $\begin{array}{l}\text { Indigestion, vomiting, loss of appetite, } \\
\text { acne, dandruff. }\end{array}$ \\
\hline $\begin{array}{l}\text { Citrus grandis (L.) } \\
\text { Osbeck. }\end{array}$ & Jaambura & Rutaceae & Fruit & Loss of appetite, fever. \\
\hline
\end{tabular}




\begin{tabular}{|c|c|c|c|c|}
\hline Botanical name & Local name & Family & Plant parts used & Uses \\
\hline Aegle marmelos (L).Corr. & Bel & Rutaceae & Fruit, Leaf, Root & Constipation, dysentery, cancer. \\
\hline $\begin{array}{l}\text { Glycosmis pentaphylla } \\
\text { (Retz.) DC. }\end{array}$ & Hotik khira & Rutaceae & $\begin{array}{l}\text { Whole plant, } \\
\text { Leaf, Stem, } \\
\text { Fruit }\end{array}$ & $\begin{array}{l}\text { Jaundice, fever, cough, anemia, eczema, } \\
\text { pimple, rheumatism, dysentery, dental } \\
\text { caries. }\end{array}$ \\
\hline Santalum album L. & Shethchondon & Santalaceae & Bark & $\begin{array}{l}\text { Tuberculosis, debility, burning sensations } \\
\text { during urination. }\end{array}$ \\
\hline Litchi chinensis Sonn. & Lichu & Sapindaceae & Seed & Indigestion, loss of appetite. \\
\hline Scoparia dulcis L. & $\begin{array}{l}\text { Modhu maloti, } \\
\text { chini pata }\end{array}$ & Scrophulariaceae & $\begin{array}{l}\text { Whole plant, } \\
\text { Leaf }\end{array}$ & $\begin{array}{l}\text { Diabetes, wounds, ulcer, weakness, fever, } \\
\text { edema, bronchitis, diarrhea, toothache. }\end{array}$ \\
\hline Smilax ovalifolia Roxb. & Koaria mul & Smilacaceae & Root & Wound. \\
\hline Solanum nigrum L. & Kakmachi & Solanaceae & Leaf, Fruit & Weakness, edema, fever, diarrhea. \\
\hline $\begin{array}{l}\text { Solanum violaceum } \\
\text { Ortega. }\end{array}$ & Bihuti & Solanaceae & Leaf, Root & $\begin{array}{l}\text { Vomiting, asthma, toothache, fever, } \\
\text { helmenthiasis. }\end{array}$ \\
\hline Datura metel fastuosa & Kal dhutura & Solanaceae & Leaf, Root, Seed & $\begin{array}{l}\text { Insanity, rheumatic swellings, abscess, } \\
\text { piles }\end{array}$ \\
\hline $\begin{array}{l}\text { Nicotiana plumbaginifolia } \\
\text { Viv.var.bigelovii Torr. }\end{array}$ & Bon tamak & Solanaceae & Root & Piles \\
\hline Cestrum nocturnum $\mathrm{L}$. & Hasna-hena & Solanaceae & Leaf & Stop bleeding. \\
\hline Datura stramonium $\mathrm{L}$. & Dhutura & Solanaceae & $\begin{array}{l}\text { Leaf, Root, } \\
\text { Seed }\end{array}$ & $\begin{array}{l}\text { Asthma, scabies, diarrhea, mental illness, } \\
\text { induce unconsciousness. }\end{array}$ \\
\hline Capsicum frutescens L. & Kacha morich & Solanaceae & Fruit & $\begin{array}{l}\text { Helmenthiasis, loss of appetite, urinary } \\
\text { disorder, indigestion. }\end{array}$ \\
\hline $\begin{array}{l}\text { Lycopersicon esculentum } \\
\text { Mill. }\end{array}$ & Tomato & Solanaceae & Whole plant & Headache, burns, induces sleep. \\
\hline Solanum melongena L. & Baegun & Solanaceae & Leaf, Fruit & Obesity, vomiting, cough, loss of appetite. \\
\hline Solanum indicum $\mathrm{L}$. & Brihoti & Solanaceae & Leaf, Stem & Rheumatism, skin diseases. \\
\hline $\begin{array}{l}\text { Solanum surattense } \\
\text { Burm.f. }\end{array}$ & Kondikari & Solanaceae & $\begin{array}{l}\text { Whole plant, } \\
\text { Leaf, Root, Seed }\end{array}$ & $\begin{array}{l}\text { Venereal diseases, asthma, chest and joint } \\
\text { pain. }\end{array}$ \\
\hline Corchorus capsularis L. & Paat & Tiliaceae & Leaf, Stem & $\begin{array}{l}\text { Constipation, stomachic, loss of appetite, } \\
\text { flatulence. }\end{array}$ \\
\hline Centella asiatica (L.) Urb. & Thankuni & Umbelliferae & $\begin{array}{l}\text { Whole plant, } \\
\text { Leaf }\end{array}$ & $\begin{array}{l}\text { Weakness, dysentery, cataract, skin } \\
\text { problem, gonorrhea, leucorrhea. }\end{array}$ \\
\hline Premna integrifolia $\mathrm{L}$. & Goniari & Verbenaceae & Leaf, Root & Fever, cold, inflammation, rheumatism. \\
\hline Gmelina arborea Roxb. & Gamir & Verbenaceae & Bark, Root & Leucorrhea, immunomodulator. \\
\hline $\begin{array}{l}\text { Clerodendrum indicum } \\
\text { (L.) Kuntze. }\end{array}$ & Bamon haki & Verbenaceae & Root & Asthma. \\
\hline $\begin{array}{l}\text { Clerodendrum viscosum } \\
\text { Vent. }\end{array}$ & Vait, Bandigach & Verbenaceae & Leaf, Root & $\begin{array}{l}\text { Snake bites, asthma, stomach pain, fever, } \\
\text { gonorrhea, leucorrhea, skin diseases. }\end{array}$ \\
\hline Cissus quadrangularis $\mathrm{L}$. & Haar-mochkanor & Vitaceae & Whole plant & $\begin{array}{l}\text { Bone fracture, rheumatic fever, } \\
\text { hemhorroid. }\end{array}$ \\
\hline Vitis quadrangularis L. & Hapta vanga & Vitaceae & $\begin{array}{l}\text { Whole } \\
\text { Plant }\end{array}$ & Rheumatic fever, joint pain. \\
\hline $\begin{array}{l}\text { Leea indica (Burm. f.) } \\
\text { Merr. }\end{array}$ & Huria & Vitaceae & Leaf, Root & $\begin{array}{l}\text { Boils, eczema, skin diseases, intestinal } \\
\text { and uterus cancer. }\end{array}$ \\
\hline $\begin{array}{l}\text { Zingiber officinale } \\
\text { Roscoe. }\end{array}$ & Ada & Zingiberaceae & Rhizome & Increase digestion. \\
\hline $\begin{array}{l}\text { Elettaria cardamomum } \\
\text { (L.) Maton. }\end{array}$ & Elachi & Zingiberaceae & Fruit & Loss of appetite. \\
\hline $\begin{array}{l}\text { Curcuma zedoaria (Berg) } \\
\text { Rosc. }\end{array}$ & Shoti & Zingiberaceae & Rhizome & Sores, stomach pain. \\
\hline Curcuma longa $\mathrm{L}$. & Kacha holud & Zingiberaceae & Rhizome & Allergy, inflammation. \\
\hline
\end{tabular}


A total of 143 plants were identified by the Kavirajes along with their medicinal uses. The plants were distributed into 62 families (Table 1). Whole plants as well as plant parts like leaf, stem, root, bark, fruit, flower, seed and gum were used for treatment. Leaves constituted the major plant part used (52\%), followed by roots $(30.55 \%)$ and whole plants (18.74\%). Other plant parts used included fruits (18.74\%), barks (13.19\%), stem (11.11\%), seeds $(11.80 \%)$ and flowers $(4.86 \%)$. The results are shown in Figure 1. Majority of the plant remedies were prepared by boiling along with crushing or pounding or soaking in cold water and concoction. But, we could not collect the data about the amount of plant extracts used by the Kavirajes as they did not want to disclose it.

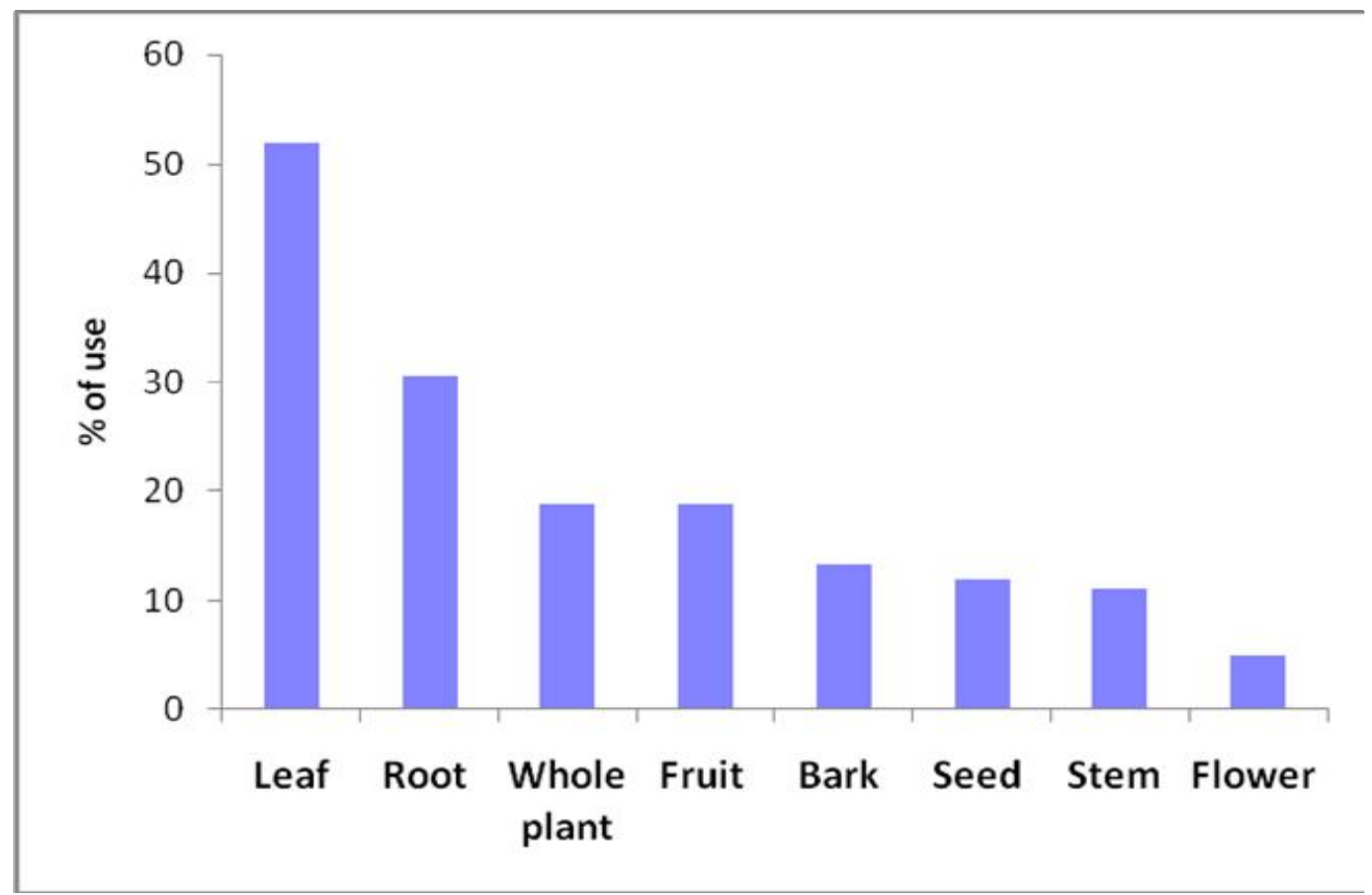

Figure 1. Percentage of use of plant parts.



Figure 2. Percentage of use of ethnomedicine in various diseases. 
About $49.30 \%$ of the plants were used to treat gastrointestinal disorders (e.g. dysentery, blood dysentery, diarrhea, stomach pain, indigestion, acidity, helminthiasis and piles) followed by respiratory tract infections (e.g. cold, coughs, asthma) (16.66\%), skin disorders (eczema, boils, itch) $(9.72 \%)$, fever (12.49\%) and sexual disorders or sexually transmitted diseases $(8.33 \%)$.

Fever, sexual disorders and urinary disorders are the other major afflictions treated by the Kavirajes. The most common disorder leucorrhea treated with 10 medicinal plants (6.95\%). However, the Kavirajes used 24 plants $(13.88 \%)$ were used for treatment of debility and 5 different plants $(3.50 \%)$ for treatment of edema, which suggests it to be a common disorder among the inhabitants of Noakhali district.

Encouragingly $15(10.41 \%)$ of the plants including Phyllanthus emblica, Citrus aurantiifolia, Allium sativum, Cocos nucifera, Momordica cochinchinensis, Azadirachta indica, Mikania cordata, Aloe barbadensis, Piper nigrum, Citrullus lanatu and Scoparia dulcis are used to treat diabetes. Three plants namely Lagenaria siceraria, Cyperus rotundus and Blumea lacera, were used to treat cholera. Piper longum, Tinospora sinensis, Santalum album, Pterocarpus santalinus were used to treat tuberculosis, Solanum indicum for the treatment of small pox and Momordica charantia, Streblus asper for the treatment of chicken pox, Clitorea tarretea and Centella asiatica for treating cataract.

Some plants were used to treat multiple diseases, while some were used as remedy for a single disease. For instance, the leaves of Tamarindus indica were used to treat chronic dysentery, rheumatic pain, cold, oral lesions. On the other hand, the leaves and flowers of Hibiscus rosasinensis were combined for treatment of leucorrhea. There were some instances where a number of plants in combination were used, for example, the whole plant of Achyranthes aspera, leaves of Centella asiatica, roots of Clerodendrum viscosum and leaves of Alternanthera sessilis are mixed together for treatment of gonorrhea, low semen, and leucorrhea. A mixture made from juice of crushed whole plant of Euphorbia prostrata, juice of whole plant of Cynodon dactylon was used for the treatment of piles. The plants Saraca asoca, Withania somnifera, Piper cubeba, Abroma augusta, and Rauwolfia serpentina were combined and administered with iron for the treatment of irregular menstruation, burning sensations in the uterus and uterine disorders. Tinospora sinensis was combined with Santalum album and Pterocarpus santalinus for the treatment of tuberculosis, debility, and burning sensations during urination.

We have also noticed some distinguished uses of several medicinal plants in these localities. For example, the leaves of Andrographis paniculata are used to cure fever, headache and vertigo in Noakhali whereas the whole plants instead of leaves are used to treat only digestion in Bagherhat district of Bangladesh. On the other hand, even the Kavirajes of Noakhali used the seed of the plant Hyptis suaveolens to treat gonorrhea whereas the Kavirajes of Khulna used the leaf, bark of this plant to treat constipation (Rahmatullah et al., 2010b).The roots of Achyranthes aspera are used in jaundice in Rajshahi, Bangladesh, while in Noakhali the whole plants are used to treat leucorrhea and gonorrhea (Nawaz et al., 2009b).These differences are quite common in the practice of traditional medicines. Different plants have different medicinal uses in different parts of Bangladesh.

\section{Conclusion}

It is thus important that modern scientific studies to be done on these medicinal plants, so that the plants may be used as remedies in a more rational and scientific manner. The results of this study revealed a rich diversity of medicinal plants used to treat various disease conditions and ethnomedicinal knowledge, amongst the residents at the various upazila in Noakhali which may through proper scientific investigations may yield novel compounds to treat both old and emerging diseases. The study should be extended to other parts of the country to discover any unknown potential use of any medicinal plants that have not been mentioned before, but is being used for the centuries to treat many difficult diseases.

\section{Acknowledgement}

The authors would like to thank the Chairman, Department of Pharmacy, Noakhali Science and Technology University, Noakhali for providing support to conduct the project work. The authors are also grateful to 
the informants and local administrators in the study area for their cooperation.

\section{References}

Akter, S., Das, P.R., Islam, M.T., Kabir, M.H., Haque, M.M., Khatun, Z., Nurunnabi, M., Khatun, Z., Lee, Y., Jahan, R. and Rahmatullah, M. 2012. A selection of medicinal plants used as blood purifiers by folk medicinal practitioners of Bangladesh. Am.-Eurasian J. Sustain. Agric. 6, 188-194.

Balick, J.M and Cox, P.A. 1996. Plants, People and Culture: the Science of Ethnobotany. Scientific American Library, New York, p. 228.

Cunningham, A.B., Ayuk, E., Franzel, S., Duguma, B. and Asanga, C., 2002. An economic evaluation of medicinal tree cultivation: Prunus africana in Cameroon. Peoples and Plants Working Paper 10 UNESCO, Paris.

Chowdhury, T., Uddin, S.B., Quraishi, D.H. and Mouri, N.J. 2011. An Ethnobotanical Survey of Plants of Sylhet in Bangladesh. Int. J. Curr. Res. 3, 31-35.

Das, P.R., Akter, S., Islam, M.T., Kabir, M.H., Haque, M.M., Khatun, Z., Nurunnabi, M., Khatun, Z., Lee, Y., Jahan, R. and Rahmatullah, M. 2012. A selection of medicinal plants used for treatment of diarrhea by folk medicinal practitioners of Bangladesh. Am.-Eurasian J. Sustain. Agric. 6, 153-161.

Das, P.R., Islam, M.T., Mahmud, A.S.M.S.B., Kabir, M.H., Hasan, M.E., Khatun, Z., Rahman, M.M., Nurunnabi, M.,Khatun, Z.,Lee,Y., Jahan,R. and Rahmatullah,M. 2012. An ethnomedicinal survey conducted among the folk medicinal practitioners of three villages in Kurigram district, Bangladesh. Am.-Eurasian J. Sustain. Agric. 6, 8596.

Esha, R.T., Chowdhury, M.R., Adhikary, S., Haque, K.M.A., Acharjee, M., Nurunnnabi, M. and Khatu Z. 2012. Medicinal plants used by tribal medicinal practitioners of three clans of the chakma tribe residing in Rangamati district, Bangladesh. Am.-Eurasian J. Sustain. Agric. 6, 7484.

Hamayun, M., Khan, M.A. and Begum, S. 2003. Marketing of medicinal plants of Utror-Gabral Valleys, Swat. Pakistan. J. Ethnobot. Leaflets SIUC USA.

Hanif, A., Hossan, M.S., Mia, M.M.K., Islam, M.J., Jahan, R. and Rahmatullah, M. 2009. Ethnobotanical survey of the Rakhain tribe inhabiting the Chittagong Hill Tracts region of Bangladesh. Am.- Eurasian J. Sustain. Agric. 3, 172-180.

Hossan, M.S., Hanif, A., Khan, M., Bari, S., Jahan, R. and Rahmatullah M.2009. Ethnobotanical survey of the Tripura tribe of Bangladesh. Am.- Eurasian J. Sustain. Agric. 3, 253-261.
Mawla, F., Khatoon, S., Rehana, F., Jahan, S., Shelley, M.M.R., Hossain, S., Haq, M., Rahman, S., Debnath, K. and Rahmatullah, M. 2012. Ethnomedicinal plants of folk medicinal practitioners in four villages of Natore and Rajshahi districts, Bangladesh. Am.-Eurasian J. Sustain. Agric. 6, 406-416.

Mia, M.M.K., Kadir, M.F., Hossan, M.S. and Rahmatullah, M. 2009. Medicinal plants of the Garo tribe Inhabiting the Madhupur forest region of Bangladesh. Am.-Eurasian J. Sustain. Agric. 3, 165-171.

Mollik, A.H., Islam. T., Khatun, A., Nasrin, D., Jahan, R. and Rahmatullah, M. 2009. Medicinal plants used against gastrointestinal tract disorders by traditional medicinal practitioners of Bangladesh. Planta Med. 75 - PD57.

Martin, G.J. 1995. 'People and Plants' Conservation Manual, Chapman and Hall, London. Ethnobotany 268.

Maundu, P. 1995. Methodology for collecting and sharing indigenous knowledge: a case study. Indigenous Knowledge and Development Monitor 3, 3-5.

Nawaz, A.H.M.M., Hossain, M., Karim, M., Khan, M., Jahan, R. and Rahmatullah, M. 2009. An ethnobotanical survey of Jessore district in Khulna division, Bangladesh. Am.Eurasian J. Sustain. Agric. 3, 195-201.

Nawaz, A.H.M.M., Hossain, M., Karim, M., Khan, M., Jahan, R. and Rahmatullah, M . 2009. An ethnobotanical survey of Rajshahi district in Rajshahi division, Bangladesh. Am.Eurasian J. Sustain. Agric. 3, 143-150.

Rahmatullah, M., Das, A.K., Haque, M., Ariful, M., Jahan, R., Khan, M.R., Chowdhury, T. and Majeedul, H. 2009. An Ethnomedicinal survey of Dhamrai Sub-district in Dhaka district, Bangladesh. Am.-Eurasian J. Sustain. Agric. 3, 881 .

Rahmatullah, M., Ferdausi, D., Mollik, M.A.H., Jahan, R., Chowdhury, M.H. and Haque, W.M. 2010. A survey of medicinal plants used by Kavirajes of Chalna area, Khulna district, Bangladesh. Afr. J. Tradit. Complement. Altern. Med. 7, 91-97.

Rahmatullah, M., Mollik, M.A.H., Paul, A.K., Jahan, R., Khatun, M.A., Seraj, S., Chowdhury, A.R., Bashar, A.B.M.A., Wahab, S.M.R. and Rahman, M.T.A. 2010. Comparative Analysis of medicinal plants used to treat Gastrointestinal Disorders in two sub-districts of Greater Khulna division, Bangladesh. Adv. Nat. Appl. Sci. 4, 22-28.

Rahmatullah, M., Momen, A., Rahman, M., Nasrin, D., Hossain, S., Khatun, Z. 2010. A randomized survey of medicinal plants used by folk medicinal practitioners in Daudkandi sub-district of Comilla district, Bangladesh. Adv. Nat. Appl. Sci. 4, 99-104. 
Rahmatullah, M., Rahman, M.A., Haque, M.Z., Mollik M.A.H., Miajee, Z.U.M.E.U., Begum, R., Rahman, M.M., Nasrin, D., Seraj, S., Chowdhury, A.R., Khatun, Z. and Khatun, M.A. 2010. A survey of medicinal plants used by Folk medicinal practitioners of Station Purbo Para Village of Jamalpur Sadar Upazila in Jamalpur district, Bangladesh. Am.-Eurasian J. Sustain. Agric. 4, 122-135.

Rahmatullah, M., Azam, M.N.K., Mollik, M.A.H., Hasan, M.M., Hassan, A.I., Jahan, R., Jamal, F., Nasrin, D., Ahmed, R., Rahman, M.M. and Khatun, M.A. 2010. Medicinal plants used by the Kavirajes of Daulatdia Ghat, Kushtia district, Bangladesh. Am.-Eurasian J. Sustain. Agric. 4, 219-229.

Rahmatullah, M., Al-Mahmud, A., Rahman, M.A., Uddin, M.F., Hasan, M., Khatun, M.A., Bashar, A.B.M.A., Ahsan, S., Mou, S.M., Begum, R. and Jahan, R. 2011. An Ethnomedicinal survey Conducted Amongst Folk medicinal practitioners in the two Southern districts of Noakhali and Feni, Bangladesh. Am.- Eurasian J. Sustain. Agric. 5, 115131.

Rahmatullah, M., Mollik, A.H., Ali, M., Abbas, F.B., Jahan, R., Khatun, A., Seraj, S. and Ahsan, S. 2011. An Ethnomedicinal Survey of Vitbilia Village in Sujanagar Sub-District of Pabna District, Bangladesh. Am.-Eurasian J. Agric. \& Environ. Sci. 10, 106-111.

Rahmatullah, M; Azam, M.N.K; Malek, I; Nasrin, D; Jamal, F; Rahman, M.A; Khatun, Z; Jahan, S; Seraj, S. and Jahan, R. 2012. An Ethnomedicinal Survey among the Marakh Sect of the Garo Tribe of Mymensingh District, Bangladesh. Int. J. Pham. Technol. Res. 4,141-149.
Rahmatullah, M., Khatun, Z., Hasan, A., Parvin, W., Moniruzzaman, M., Khatun, A., Mahal, M.J., Shariful, M., Bhuiyan, A., Mou, S.M. and Jahan, R. 2012. Survey and Scientific evaluation of medicinal plants used by the Pahan and Teli Tribal Communities of Natore district, Bangladesh. Afri. J. Tradit. Complement. Alter. Med. 9, 366-73.

Rana, M.P., Sohel, M.I.S., Akhter, S. and Islam, M.J. 2010. Ethnomedicinal plants use by the Manipuri tribal community in Bangladesh J. Forest. Res. 21, 85-92.

Shahidullah, M., Al-Mujahidee, M., Uddin, S.M. N., Hossan, M.S., Hanif, A., Bari, S. and Rahmatullah, M. 2009. Medicinal plants of the Santal tribe residing in Rajshahi district, Bangladesh. Am.- Eurasian J. Sustain. Agric. 3, 220-226.

Uddin, S.N., Uddin, M.Z., Hassan, M.A. and Rahman, M.M. 2004. Preliminary ethnomedical plant survey in Khagrachari district, Bangladesh. Bangladesh J. Plant Taxon. 11, 39-48.

Uddin, M.Z., Hassan, M.A. and Sultana, M. 2006. Ethnobotanical survey of medicinals plants in Phulbari Upazilla of Dinazpur District, Bangladesh. Bangladesh J. Plant Taxon. 13, 63-68.

WHO: Traditional medicine 2003. Fact sheet No 134. 